\section{Robotic minimally invasive cell transplantation for heart failure}

\author{
Harald C. Ott, MD, ${ }^{a}$ Johannes Brechtken, MD, ${ }^{b}$ Cory Swingen, PhD, ${ }^{\mathrm{c}}$

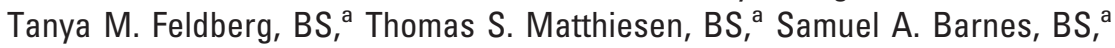 \\ Wendy Nelson, PhD, and Doris A. Taylor, PhD, ${ }^{a}$ Minneapolis, Minn
}

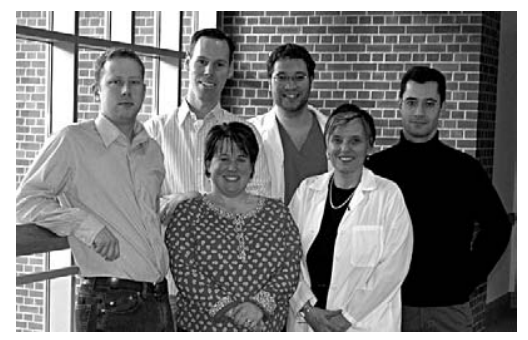

Drs Swingen, Matthiesen, Nelson, Ott, Taylor, and

ardiac cell transplantation offers new opportunities as a potent therapeutic tool to improve left ventricular $(\mathrm{LV})$ function and reverse postinfarction remodeling in ischemic heart disease. Skeletal myoblasts (SKMBs) engraft within infarcted myocardium, form myotubes, induce angiogenesis, and improve both diastolic and systolic LV function. ${ }^{1}$ Bone marrow-derived mononuclear cells (BM-MNCs) likewise engraft, increase angiogenesis, and improve myocardial perfusion. $^{2}$ Both cell types have moved to clinical testing, and preclinical studies suggest that they could have synergistic functional benefits that argue for combined transplantation. ${ }^{3,4}$ Intramyocardial injections are currently performed either percutaneously through an endoventricular or transvenous approach or surgically through a thoracotomy or sternotomy. We recently reported a video-assisted thoracoscopic technique to reduce invasiveness and perioperative risk of surgical cell delivery that was tested in uninjured swine hearts. ${ }^{5}$ In the setting of heart failure (HF), mechanical manipulation of the left ventricle both by means of stabilization and cell injection must be minimized to prevent hemodynamic compromise, arrhythmia, and ventricular perforation. Robotically assisted cardiac surgery combines the advantages of minimal invasiveness and thoracoscopic access but adds a 3-dimensional view and 7 degrees of freedom that requires less cardiac manipulation than with the 2-dimensional view and limited freedom of motion of video-assisted thoracoscopic surgery. ${ }^{6} \mathrm{We}$ therefore propose a robot-assisted, beating-heart cell transplantation technique for use in severe HF to increase safety, optimize targeting, and reduce procedural time.

\section{Procedure Description}

Eleven injured swine in which HF was previously induced by means of coronary occlusion and coronary embolism (left anterior

From the Center for Cardiovascular Repair, ${ }^{a}$ the Division of Cardiology, and the Department of Radiology, ${ }^{\mathrm{c}}$ University of Minnesota, Minneapolis, Minn.

This work was supported in part by National Heart, Lung, and Blood Institute/National Institutes of Health awards to Dr Taylor (R-01 HL-63346, HL-63703).

Received for publication Nov 19, 2005; revisions received Feb 1, 2006; accepted for publication Feb 21, 2006.

Address for reprints: Doris A. Taylor, PhD, Center for Cardiovascular Repair, University of Minnesota, 312 Church St SE, BSBE 7, Minneapolis, MN 55455 (E-mail: dataylor@umn.edu).

J Thorac Cardiovasc Surg 2006;132:170-3

$0022-5223 / \$ 32.00$

Copyright @ 2006 by The American Association for Thoracic Surgery doi:10.1016/j.jtcvs.2006.02.017 Brechten (left to right)

descending coronary artery, $\mathrm{n}=9$; circumflex artery, $\mathrm{n}=2$ ) underwent robot-assisted cell $(n=7)$ or vehicle $(n=4)$ injection by using the daVinci robotic system (Intuitive Surgical, Sunnyvale, Calif). During right single-lung ventilation and antiarrhythmic prophylaxis (amiodarone, $3 \mathrm{mg} / \mathrm{kg}$; lidocaine, $1 \mathrm{mg} / \mathrm{kg}$ ), we inserted the camera port, 2 instrument ports, and an auxiliary port (Figure 1, A). After removal of the pericardial fat pad, we incised the pericardium along the sternal border, dissected pericardial adhesions, and created a triangular pericardial flap. We inserted the prefilled injection needle (27-gauge needle attached to 12-inch tubing; Saf-T E-Z Set, BD, Sandy, Utah) through the auxiliary port and injected a 7-mL cell suspension containing a combination of $2.9 \times 10^{8} \pm 5.9 \times 10^{7}$ autologous SKMBs and $1.1 \times 10^{8} \pm 6.8$ $\times 10^{6}$ autologous BM-MNCs (Figure $1, B$ ) at 6 to 10 sites. SKMBs were iron oxide labeled, as previously described. ${ }^{5}$ Viability at the time of injection was greater than 85\%, and CD56 expression was greater than $80 \%$. BM-MNCs were acutely isolated from bone marrow aspirate through Ficoll density gradient centrifugation. Injections were performed tangentially to minimize perforation risk and injectate backflow, covering a target area of 15 to $20 \mathrm{~cm}^{2}$. After cell delivery, the pericardial flap was readapted, all instrument ports were removed, and the left lung was expanded under visual control. We inserted a chest tube through the inferior instrument port, closed the port sites in 3 layers, and flushed the left thoracic cavity with $0.9 \%$ saline $(200 \mathrm{~mL})$. After removal of the chest tube, animals were extubated and recovered according to postoperative standards. Baseline magnetic resonance imaging (MRI) was performed 5 weeks after myocardial injury. Follow-up MRI was repeated at 4 and 7 weeks after cell/vehicle transplantation.

\section{Results}

Cell transplantation was completed successfully in 6 of 7 cases. Intractable ventricular fibrillation occurred in one animal during cell injection. No conversion to open chest surgery was necessary, and no other procedure-related complications occurred. Over the course of the study, single-lung ventilation time was reduced to a minimum of 23 minutes, and total anesthesia time was reduced to a minimum of 44 minutes. Cells were successfully transplanted into the apical, anterior, and lateral target regions of the left ventricle, including into thinned sections of the scar (target region wall thickness, 3-14 mm), without ventricular perforation. Postoperative MRI studies confirmed retention of iron oxide-labeled cells in the apex (Figure 2, A) and lateral wall (Figure 2, B) up to 7 weeks after injection. Prussian blue staining of tissue sections showed engraftment of iron oxide-labeled myotubes in treated areas (Figure 2, C). Immunofluorescent staining for slow skeletal 

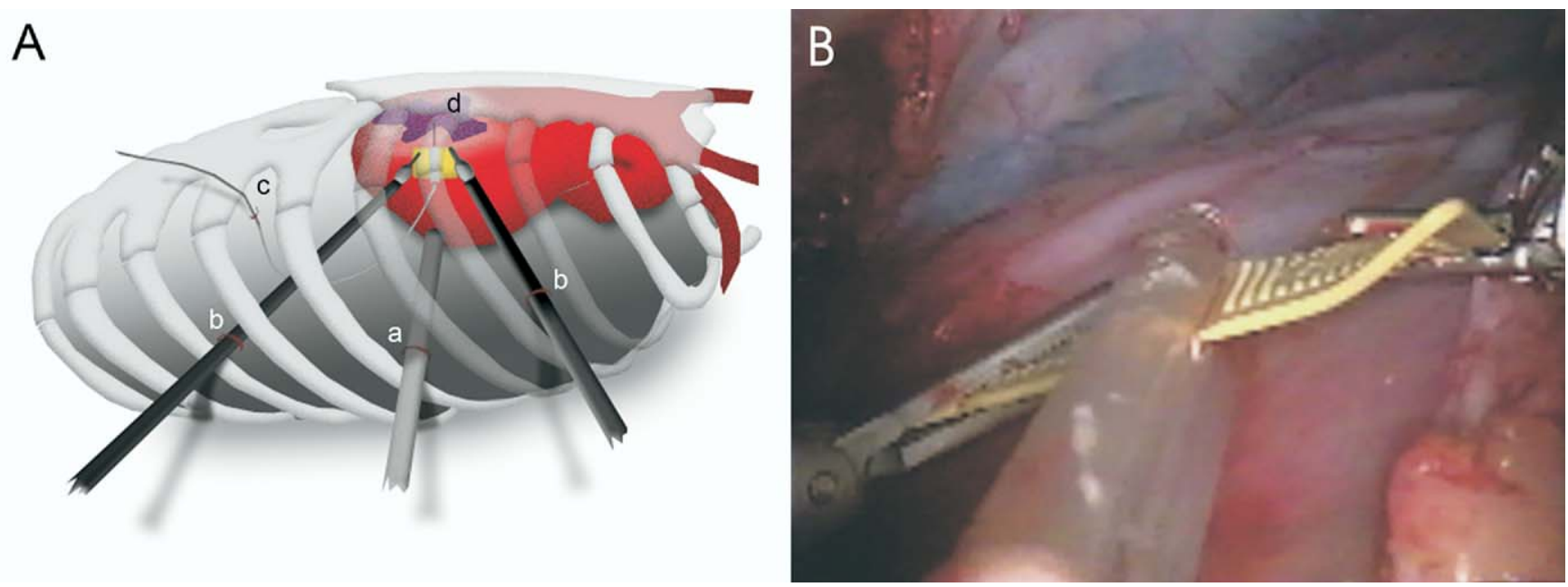

Figure 1. A, Schematic of robotic cell transplantation using the daVinci surgical robot. Swine are placed in the supine position and slightly rotated to the right, with elevated shoulders to expand the intercostal spaces. The camera port $(a)$ is placed in the fifth, instrument ports $(b)$ are placed in the third and seventh, and the auxiliary port is placed in the sixth (c) intercostal spaces. The 27-gauge winged injection needle (d) is inserted through the auxiliary port, allowing multiple tangential injections into the target area (shown in purple). B, Intrathoracic view of the injection device before and during intramyocardial injection. During injection, the rubber wings of the needle allow safe guidance but minimize the mechanical force applied to the left ventricular wall during slow injection.

myosin confirmed the skeletal muscle phenotype (Figure 2, D). Functional data are shown in Table 1.

\section{Discussion}

Within the past few years, robot-assisted surgery has revolutionized minimally invasive cardiac surgery, allowing complex procedures to be performed on the beating heart. Its major advantages are increased degrees of freedom, 3-dimensional vision, and magnification of the operating field. Furthermore, it combines minimally invasive access with improved targeting and a decreased risk of hemodynamic compromise and ventricular perforation, making it amenable to patients with severely impaired LV function and remodeling who are not necessarily amenable to endoscopic pro- cedures. In the present study the robot-assisted technique allowed controlled cell delivery to the anterior, apical, and lateral regions of the left ventricle, with minimal requirement for stabilization and mechanical manipulation. In contrast to current catheter-based transventricular approaches, safe treatment of target segments with a wall thickness of less than $5 \mathrm{~mm}$ was achievable. Although efficacy was a secondary end point of the present study, the improved LV function after cell delivery reinforces the feasibility of this procedure. A more thorough investigation, including larger experimental groups, will be necessary to clarify whether the herein proposed combination of autologous SKMBs and BMMNCs is superior to the isolated transplantation of each cell type. If this is the case, as suggested by other preclinical studies, ${ }^{3,4}$ our

Table 1. Functional measurements from contrast-enhanced magnetic resonance imaging studies performed 5 weeks after myocardial injury (baseline) and 4 and 7 weeks after cell or vehicle transplantation

\begin{tabular}{|c|c|c|c|c|c|c|c|c|c|}
\hline & \multicolumn{3}{|c|}{ MRI baseline } & \multicolumn{3}{|c|}{ MRI wk 4} & \multicolumn{3}{|c|}{ MRI wk 7} \\
\hline & Cell treated & Control & $P$ value & Cell treated & Control & $P$ value & Cell treated & Control & $P$ value \\
\hline EF (\%) & $33.25 \pm 10.14$ & $36.67 \pm 4.39$ & .573 & $36.75 \pm 9.94$ & $33.54 \pm 4.57$ & 639 & $39.07 \pm 5.24$ & $30.1 \pm 6.76$ & .040 \\
\hline RWM (mm) & $3.64 \pm 1.16$ & $3.75 \pm 1.85$ & .884 & $6.06 \pm 1.60$ & $2.96 \pm 1.13$ & .001 & $6.40 \pm 1.71$ & $4.05 \pm 1.57$ & .029 \\
\hline WTHGN (\%) & $18.24 \pm 14.49$ & $15.05 \pm 12.53$ & .586 & $21.69 \pm 16.64$ & $6.60 \pm 5.35$ & .037 & $29.69 \pm 14.61$ & $8.93 \pm 7.87$ & .020 \\
\hline LVEDV (ml) & $193.03 \pm 13.61$ & $190.65 \pm 11.67$ & .830 & $241.1 \pm 9.7$ & $244.1 \pm 9.1$ & .698 & $269.38 \pm 12.33$ & $298.23 \pm 13.70924$ & .031 \\
\hline
\end{tabular}

MRI studies were performed with a 1.5-T clinical scanner (Siemens Sonata; Siemens AG, Munich, Germany). Electrocardiography-gated cine MR images were acquired of the entire cardiac cycle in approximately 16 to 20 phases by using a steady-state free precession pulse sequence in both long- and short-axis orientations covering the entire left ventricle. Values are expressed as means \pm standard deviation. Comparisons between cell-treated and control groups were performed with the unpaired Student $t$ test. MRI, Magnetic resonance imaging; $E F$, ejection fraction; RWM, regional wall motion; WTHGN, wall thickening; LVEDV, left ventricular end-diastolic volume. 

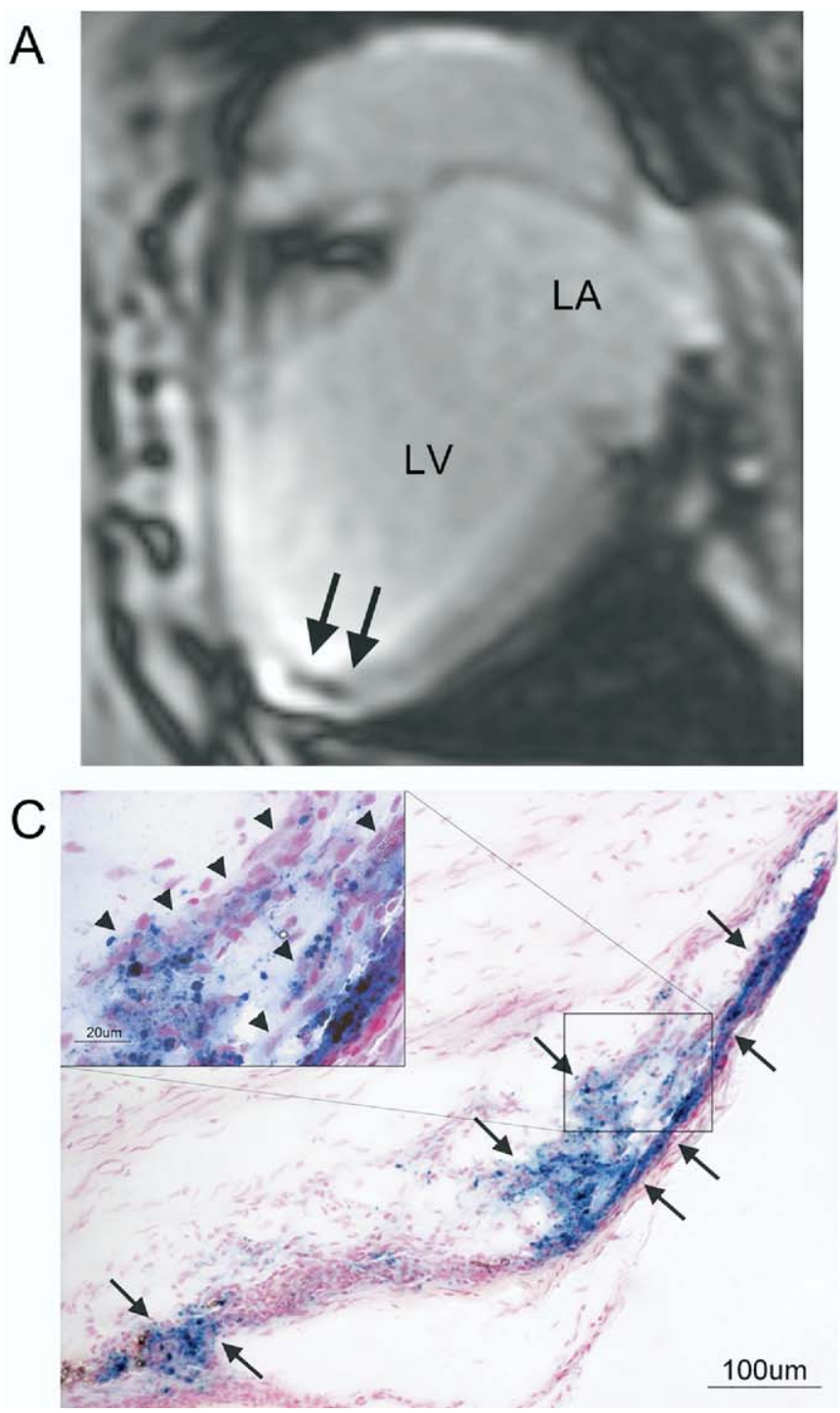

B

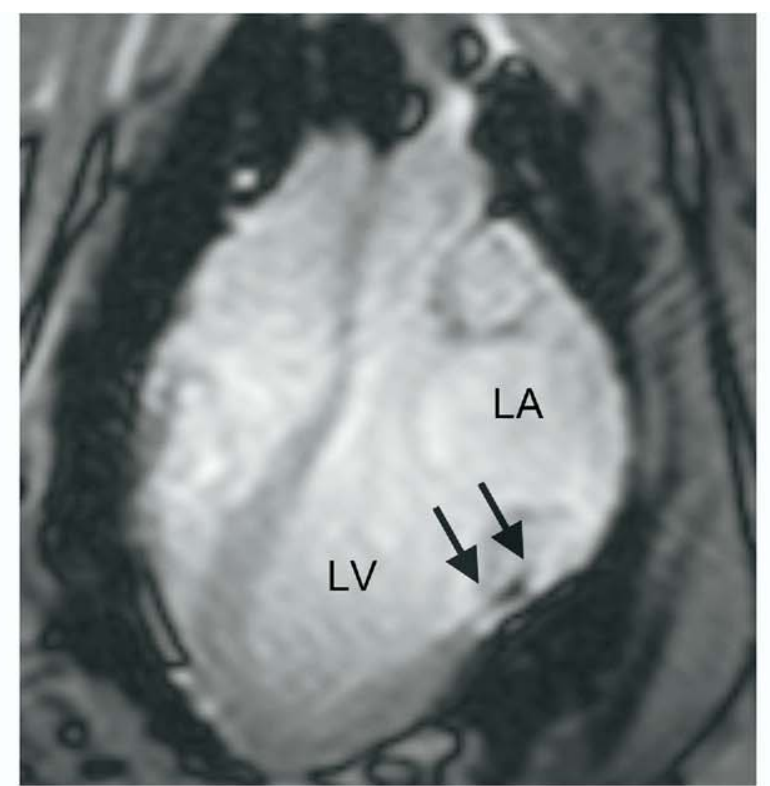

D

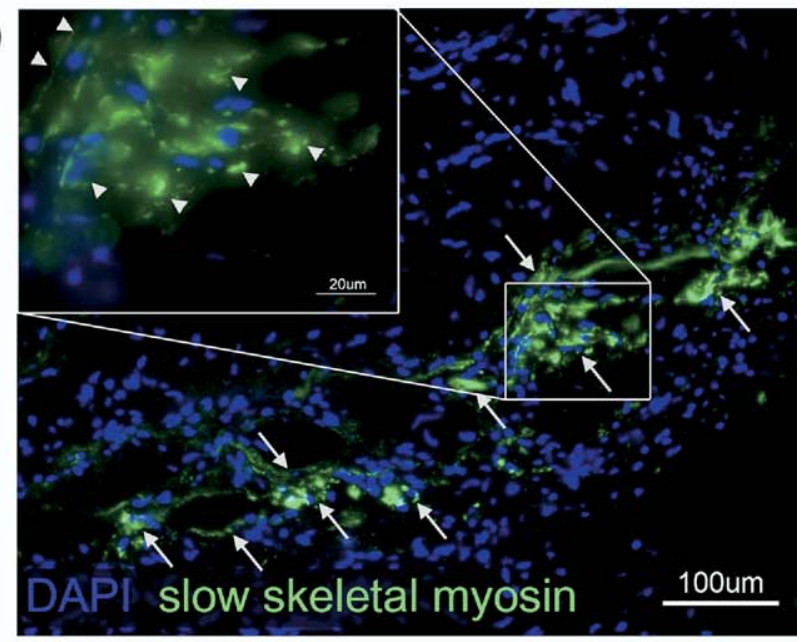

Figure 2. Upper panels, Contrast-enhanced magnetic resonance cine images showing long-axis views of 2 cell-treated hearts. Cells are visible as signal voids (black dots) in the contrast-enhanced (shown in white) apical region (A) and the lateral wall (B) of the left ventricle (white arrows). $L V$, Left ventricle; $L A$, left atrium. Lower panels, Corresponding histologic sections of a cell-treated region at low and high magnification. C, Engrafted iron oxide-labeled myoblasts within the fibrotic infarction scar stain with Prussian blue (black arrows and arrowheads). D, Immunofluorescent staining for slow skeletal myosin (shown in green) shows the expression of skeletal muscle proteins in engrafted cells (white arrows and arrowheads), suggesting cell differentiation. Nuclei within the section are evidenced by fluorescent blue 4',6-Diamidino-2-phenylindole (DAPI) staining.

proposed protocol would offer a streamlined option to provide patients with HF a combination of autologous SKMBs and BMMNCs in one minimally invasive procedure.

In summary, this new method might provide a feasible option for patients who are currently not eligible for surgical cell transplantation. It combines the benefits of a surgical approach with the reduction of perioperative risk associated with a minimally inva- sive procedure. Further investigation is required to clarify whether the efficacy of robotic cell transplantation equals direct surgical and catheter-based injection.

We thank experimental surgical services and research animal resources at the University of Minnesota for diligent and expert assistance in animal anesthesia and perioperative care. 


\section{References}

1. Taylor DA, Atkins BZ, Hungspreugs P, Jones TR, Reedy MC, Hutcheson KA, et al. Regenerating functional myocardium: improved performance after skeletal myoblast transplantation. Nat Med. 1998;4: 929-33.

2. Orlic D, Kajstura J, Chimenti S, Jakoniuk I, Anderson SM, Li B, et al. Bone marrow cells regenerate infarcted myocardium. Nature. 2001;410: 701-5.

3. Ott HC, Bonaros N, Marksteiner R, Wolf D, Margreiter E, Schachner T, et al. Combined transplantation of skeletal myoblasts and bone marrow stem cells for myocardial repair in rats. Eur J Cardiothorac Surg. 2004;25:627-34.

4. Memon IA, Sawa Y, Miyagawa S, Taketani S, Matsuda H. Combined autologous cellular cardiomyoplasty with skeletal myoblasts and bone marrow cells in canine hearts for ischemic cardiomyopathy. J Thorac Cardiovasc Surg. 2005;130:646-53.

5. Thompson RB, Parsa CJ, van den Bos EJ, Davis BH, Toloza EM, Klem I, et al. Video-assisted thoracoscopic transplantation of myoblasts into the heart. Ann Thorac Surg. 2004;78:303-7.

6. Ott HC, Bonatti J, Mueller DL, Chevtchik O, Riha M, Danzmeyr M, et al. Robotically enhanced cardiac surgery. Eur Surg. 2002;34:183-9.

\title{
Percutaneous closure of a ventricular septal defect after surgical treatment of hypertrophic cardiomyopathy
}

\author{
D. De Wolf, MD, PhD, ${ }^{a}$ Y. Taeymans, MD, PhD, ${ }^{b}$ B. Suys, MD, ${ }^{\mathrm{c}}$ D. Matthys, MD, PhD, ${ }^{a}$ and G. Van Nooten, MD, PhD, ${ }^{d}$ \\ Ghent and Antwerp, Belgium
}

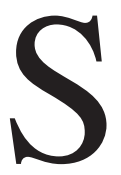

urgery or ethanol ablation is indicated in patients with hypertrophic cardiomyopathy who have symptomatic New York Heart Association (NYHA) class III disease with severe left ventricular outflow tract (LVOT) gradients despite medical treatment. ${ }^{1}$ A ventricular septal defect (VSD) after surgical treatment is rare. ${ }^{2}$ Alternatives for surgical closure are available in patients with congenital VSDs or post-myocardial infarction VSDs. Percutaneous closure provides a reasonable alternative. $^{3,4}$

We describe the technique of percutaneous closure of a VSD after surgery for hypertrophic cardiomyopathy. ${ }^{5}$

\section{Clinical Summary}

A 50-year-old man was referred for surgery with symptomatic hypertrophic cardiomyopathy despite medical therapy. He was in NYHA class III with effort-related angina. Echocardiography showed asymmetrical left ventricular hypertrophy with a septal end-diastolic thickness of $19 \mathrm{~mm}$, systolic anterior motion, an ejection fraction of $66 \%$, and an LVOT gradient of $64 \mathrm{~mm} \mathrm{Hg}$. During catheterization the pressure gradient measured $100 \mathrm{~mm} \mathrm{Hg}$. Ethanol ablation failed. A surgical procedure was performed via a transaortic approach. By a Morrow septal myomectomy the LVOT was increased from 16 to $23 \mathrm{~mm}$. However, on control transesophageal echocardiography the distal part of the LVOT was still

From the Departments of Congenital Cardiology, ${ }^{\mathrm{a}}$ Cardiology, ${ }^{\mathrm{b}}$ and Cardiac Surgery, ${ }^{\mathrm{d}}$ Ghent University Hospital, Ghent, Belgium, and the Department of Congenital Cardiology, ${ }^{\mathrm{c}}$ Antwerp University Hospital, Antwerp, Belgium.

Received for publication Dec 23, 2005; revisions received Jan 4, 2006; accepted for publication Jan 10, 2006.

Address for reprints: Daniël De Wolf, MD, PhD, Kindercardiologie UZ Gent, De Pintelaan 185, 9000 Gent, Belgium (E-mail: Daniel.dewolf@ugent.be).

J Thorac Cardiovasc Surg 2006;132:173-4

$0022-5223 / \$ 32.00$

Copyright $\odot 2006$ by The American Association for Thoracic Surgery doi:10.1016/j.jtcvs.2006.01.051 obstructive. This part being out of reach, a second attempt was made via the left atrium during the same procedure. The anteroexternal commissure and the A1 and A2 portions of the anterior leaflet of the mitral valve were detached from the annulus, and the myomectomy was completed distally. The anterior leaflet of the mitral valve was reattached to the annulus. Control echocardiography showed total relief of the LVOT obstruction, absence of mitral regurgitation, but a small muscular VSD. The initial postoperative course was uneventful. During the following weeks, the patient's clinical condition deteriorated, with left and right ventricular failure and mild renal and hepatic failure. On control echocardiography a muscular VSD $12 \mathrm{~mm}$ in diameter with a left-to-right shunt of 2:1 and half systemic right ventricular pressure was seen. At the right side of the ventricular septum, the VSD was located near the attachments of the septal leaflet of the tricuspid valve; at the left side, the distance from the aortic valve was $18 \mathrm{~mm}$. The thickness of the septum surrounding the VSD varied between 10 and $14 \mathrm{~mm}$.

On the 20th postoperative day, percutaneous closure of the VSD was attempted. Under fluoroscopy and transesophageal echocardiographic guidance, the VSD could be crossed via the right jugular vein and a Mullins transseptal sheath was positioned in the left ventricle. An Amplatzer 14-mm VSD occluder (AGA Medical Corporation, Golden Valley, Minn) was delivered in the VSD and released (Figure 1). The patient had a second-degree atrioventricular block necessitating temporary pacing. Hemodynamic recovery was immediate, but the postoperative course was complicated by transient hemolysis and Candida septicemia. One month after closure of the VSD, the patient could be dismissed. An echocardiogram showed a correct position of the VSD device, without any gradient or residual shunt across the LVOT (Figure 2).

\section{Discussion}

A VSD complicating surgery for LVOT obstruction in hypertrophic cardiomyopathy is rare. ${ }^{2}$ These VSDs can be hemodynamically significant. Surgical closure of a VSD can be cumbersome in a patient who has had recent surgery. Percutaneous or perventricu- 